\title{
Glycosylation modulates TRAIL-R1/death receptor 4 protein: Different regulations of two pro-apoptotic receptors for TRAIL by tunicamycin
}

\author{
TATSUSHI YOSHIDA $^{1}$, TAKUMI SHIRAISHI ${ }^{1,2}$, MANO HORINAKA $^{1}$, MIKI WAKADA $^{1}$ and TOSHIYUKI SAKAI $^{1}$ \\ ${ }^{1}$ Department of Molecular-Targeting Cancer Prevention, ${ }^{2}$ Department of Urology, Graduate School of Medical Science, \\ Kyoto Prefectural University of Medicine, Kawaramachi-Hirokoji, Kamigyo-ku, Kyoto 602-8566, Japan
}

Received May 3, 2007; Accepted July 27, 2007

\begin{abstract}
Death receptor 4 (DR4) is a receptor of the antitumor death ligand, TNF-related apoptosis-inducing ligand (TRAIL), and is considered a promising molecular target for cancer therapy. Here, we show a novel regulation of DR4 protein. Tunicamycin treatment, which is an inducer of endoplasmic reticulum (ER)-stress, generated a lower molecular-weight pattern of DR4, but not DR5 protein in prostate cancer DU145 and PC3 cells. Thus, we termed the small form of DR4 protein, DR4-Small (DR4-S) and the large form, DR4-Large (DR4-L). Using DR4 siRNA, we confirmed that DR4-S also stands for DR4 protein. Other ER-stress inducers, brefeldin A and thapsigargin did not generate DR4-S. On the other hand, these ER-stress inducers increased DR5 protein. Tunicamycin induces ER-stress following the inhibition of $\mathrm{N}$-linked glycosylation. Thus, we examined DR4 protein in cell lysates treated with glycosydase. Glycosydase treatments generated DR4-S protein, similar to tunicamycin. These results indicate that tunicamycin regulates DR4 protein size via inhibition of glycosylation.
\end{abstract}

\section{Introduction}

TNF-related apoptosis-inducing ligand (TRAIL) $(1,2)$ induces apoptosis selectively in cancer cells in vitro and in vivo, with little or no toxicity in normal cells (3-5); therefore, TRAIL is one of the most promising new candidates for cancer therapeutics and has been applied to a phase I clinical trial. TRAIL has two specific pro-apoptotic receptors, death receptor 4 and 5 (DR 4 and 5 also called TRAIL-R1 and -R2,

Correspondence to: Dr Toshiyuki Sakai, Department of MolecularTargeting Cancer Prevention, Graduate School of Medical Science, Kyoto Prefectural University of Medicine, Kawaramachi-Hirokoji, Kamigyo-ku, Kyoto 602-8566, Japan

E-mail: tsakai@koto.kpu-m.ac.jp

Abbreviations: DR4, death receptor 4; TRAIL, tumor necrosis factor-related apoptosis-inducing ligand; ER, endoplasmic reticulum

Key words: DR4, glycosylation, TRAIL, tunicamycin, DR5 respectively) (6-10). TRAIL binding to DRs results in the formation of death-inducing signaling complex (DISC), containing Fas-associated death domain (FADD) and procaspase- 8 , which leads to the cleavage and activation of procaspase- $8(9,10)$. Consequently, activated caspase- 8 triggers apoptosis via the activation of effector caspase-3 and -7 .

Recently, agonistic antibodies against DR4 and 5, which can substitute for the TRAIL function, have been developed $(11,12)$. In particular, phase I and II clinical studies have been initiated with the DR4 antibody, HGS-ETRI, against solid tumors (13). Thus, DR4 is a promising molecular target for cancer therapy; however, the regulation of DR4 has not been fully elucidated.

$\mathrm{N}$-linked oligosaccharides are added cotranslationally to newly synthesized polypeptides in the endoplasmic reticulum (ER) (14). These glycans are subjected to extensive modification as mature glycoproteins through the ER via the Golgi complex. N-linked glycosylation plays a role in the essential function of matured glycoproteins. A member of ectonucleoside triphosphate diphosphohydrolase 1, CD39, is inactive in intracells; however, glycosylated CD39 is localized at the cell surface and has activity (15). N-glycosylation in the granulocyte-macrophage colony-stimulating factor receptor $\alpha$ subunit is essential for ligand binding and signaling (16). Moreover, a lack of fucose of N-linked oligosaccharide on human IgG1 improves binding to Fc receptor III and antibody-dependent cellular toxicity (17).

We previously reported that tunicamycin, which is an antibiotic and an inducer of ER-stress following the inhibition of N-linked glycosylation (18), up-regulated DR5 expression and sensitized cancer cells to TRAIL-induced apoptosis (19). Here, we examined the effect of tunicamycin on DR4 and found that tunicamycin caused a lower-molecular weight pattern of DR4 protein. In addition, we demonstrated that the modulation depended on the inhibition of glycosylation. In this study, we report a novel regulation of DR4 protein related to glycosylation.

\section{Materials and methods}

Reagents. Tunicamycin, brefeldin A and thapsigargin were purchased from Sigma Chemical Co. (St. Louis, MO) and dissolved in DMSO. 
Cell culture. Human prostate cancer DU145 and PC3 cells were maintained in RPMI-1640 medium supplemented with $10 \%$ fetal bovine serum, $100 \mathrm{U} / \mathrm{ml}$ penicillin and $100 \mu \mathrm{g} / \mathrm{ml}$ streptomycin, and incubated at $37^{\circ} \mathrm{C}$ in a humidified atmosphere of $5 \% \mathrm{CO}_{2}$.

Western blot analysis. Cell lysate was prepared as described previously (20). Cell lysate containing $50 \mu \mathrm{g}$ protein was separated on $10 \%$ SDS-polyacrylamide gel for electrophoresis, and blotted onto polyvinylidene difluoride (PVDF) membranes (Millipore, Bedford, MA). Rabbit polyclonal anti-DR5 (Cayman Chemical, Ann Arbor, MI) and anti-DR4 antibodies (ProSci, Poway, CA) and mouse monoclonal anti- $\beta$-actin antibody (Sigma Chemical, St Louis, MO) were used as the primary antibodies. The signal was detected with an ECL Western blot analysis system (Amersham Pharmacia Biotech, Piscataway, NJ). Prestained protein marker was purchased from New England Biolabs (Beverly, MA).

Small interfering RNA. The DR4 and control LacZ siRNA sequences used were previously described $(21,22)$. One day prior to transfection, cells were seeded without antibiotics to a density of $1 \times 10^{5}$ cells/well on 6-well plates. DR4 and LacZ siRNA $(20 \mathrm{nM})$ were transfected into cells using a modified oligofectamine protocol (Invitrogen, Carlsbad, CA), for which the volume of oligofectamine was reduced to one-third the recommended volume to limit toxic effects.

Glycosydase treatment. PNGase F and Endo Hf were purchased from New England Biolabs. Whole cell lysates prepared from DU145 or PC3 cells were treated with PNGase F or Endo Hf, following the protocols by the manufacturer.

\section{Results}

Tunicamycin generates a lower-molecular weight band pattern of DR4 protein. We previously reported that tunicamycin treatment induces DR5 expression in human prostate cancer DU145 cells (19). Next, we examined the effect of tunicamycin on DR4 protein. As shown in Fig. 1A, DR4 protein migrated toward a lower-molecular weight position by tunicamycin treatment in a dose-dependent manner. On the other hand, another TRAIL receptor DR5 protein did not change, but increased by tunicamycin treatment as we reported previously (19). We termed the small form of DR4 protein DR4-Small (DR4-S) and the large form DR4-Large (DR4-L). DR4-S caused by tunicamycin was observed in other prostate cancer PC3 cells as well as DU145 cells, indicating that the modulation of DR4 protein is not cell line-specific. To examine whether DR4-S includes DR4 protein, we employed DR4 siRNA. Knockdown of DR4 expression by siRNA reduced both DR4-L and DR4-S detected with anti-DR4 antibody (Fig. 1B). DR4 siRNA did not change DR5 and $B$-actin proteins, indicating that siRNA specifically reduced DR4 expression. These results indicate that DR4-S is also DR4 protein.

Thapsigargin and brefeldin A do not generate DR4-S protein. As tunicamycin is an inducer of ER-stress (18), we hypothesized that ER-stress might generate DR4-S protein. We
(A)

\section{DU145}

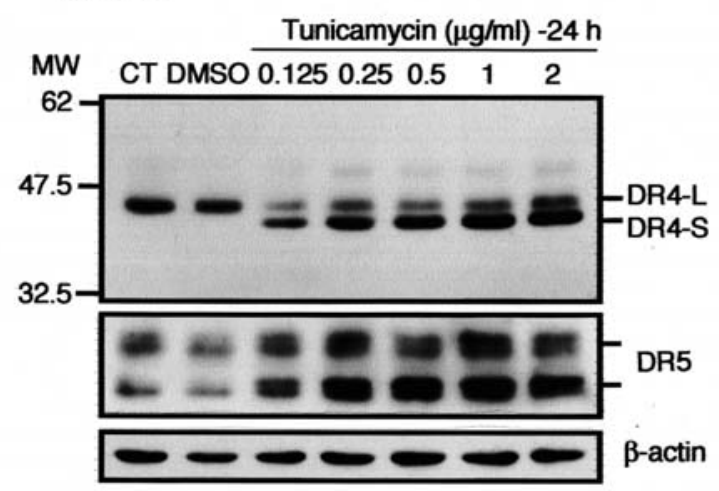

PC3

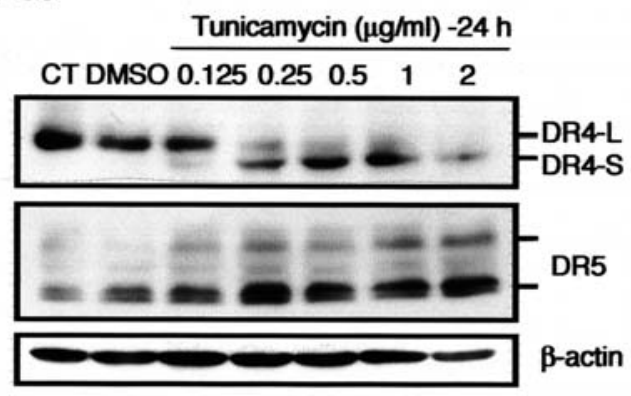

(B)

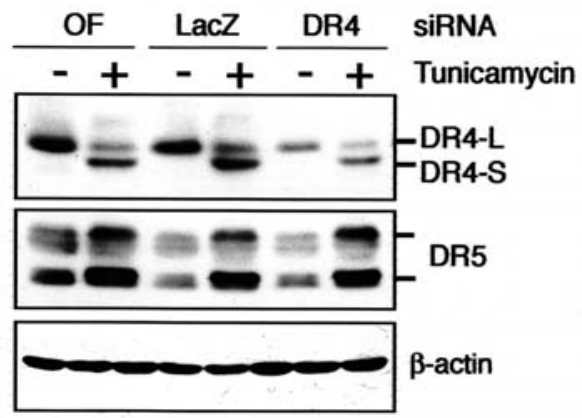

Figure 1. (A) Tunicamycin shifts DR4 protein to lower-molecular weight band position. DU145 and PC3 cells were treated with indicated concentrations of tunicamycin for $24 \mathrm{~h}$. Whole cell extracts were resolved by Western blotting with anti-DR4, DR5 and B-actin antibodies. B-actin is a loading control. $\mathrm{CT}$, treated with medium only. DMSO, treated with medium containing solvent. Molecular weight markers are indicated. (B) DR4-S protein caused by tunicamycin is reduced by DR4 siRNA. After transfection of DR4 siRNA, DU145 cells were treated with $2 \mu \mathrm{g} / \mathrm{ml}$ tunicamycin for $24 \mathrm{~h}$. Western blotting was carried out with anti-DR4, DR5 and B-actin antibodies. -, treated with solvent DMSO. OF, treated with transfection reagent oligofectamin only. LacZ, transfected with control LacZ siRNA.

investigated the effects of other ER-stress inducers, thapsigargin (23) and brefeldin A (24) on DR4 protein. As shown in Fig. 2, neither agent modulated DR4 protein. On the other hand, both agents increased DR5 protein in a dose-dependent manner.

DR4-S is caused by inhibition of glycosylation. Tunicamycin, thapsigargin and brefeldin A are inducers of ER-stress; however, the mechanisms of ER-stress induction are different. Tunicamycin inhibits N-linked glycosylation of proteins followed by ER-stress (18). Thapsigargin releases intracellular 

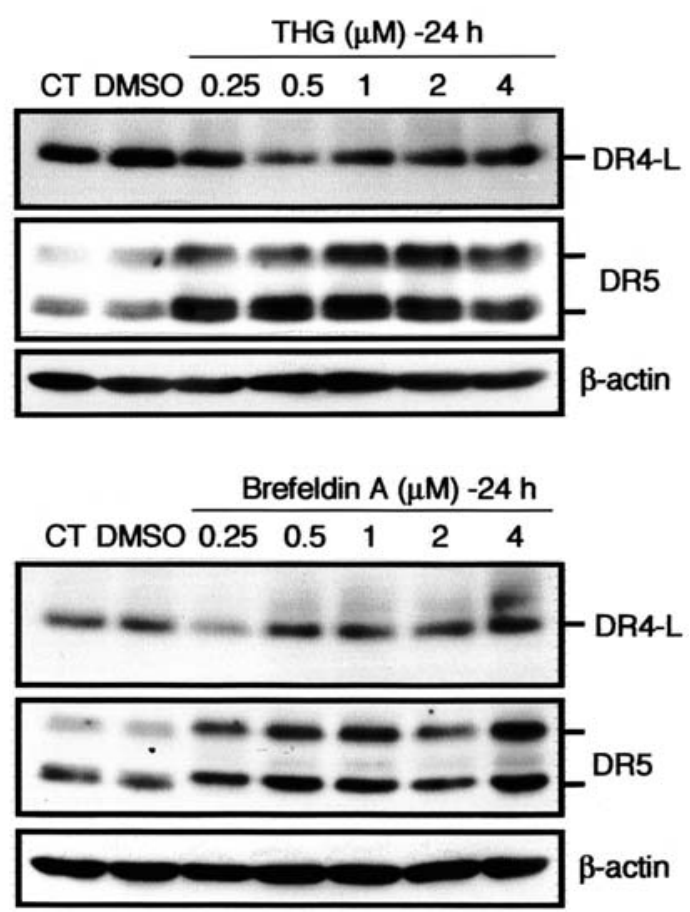

Figure 2. Other ER-stress inducers, thapsigargin and brefeldin A, do not change DR4 protein. DU145 cells were treated with indicated concentrations of thapsigargin or brefeldin A for $24 \mathrm{~h}$. Western blotting was carried out with indicated antibodies. B-actin is a loading control. CT, medium change only. THG, thapsigargin.

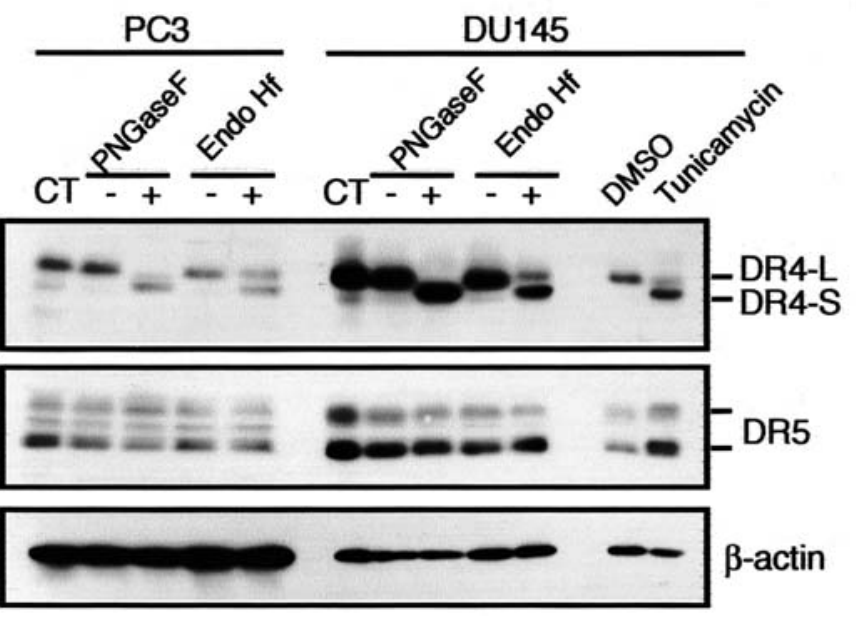

Figure 3. Inhibition of glycosylation causes DR4-S protein. Whole cell lysates of DU145 and PC3 cells were treated with PNGaseF and Endo Hf. After treatment, the cell lysates were resolved by Western blotting with indicated antibodies. ß-actin is a loading control. CT, medium change only. - , treated with recommended buffer without enzyme.

calcium and consequently induces ER-stress (23). Brefeldin A induces ER-stress following disruption of the structure and function of the Golgi apparatus (24). Thus, we considered that the modulation of DR4 protein by tunicamycin might be caused by the inhibition of glycosylation rather than ER-stress. Next, we carried out Western blotting of DR4 using cell lysates treated with glycosydases, PNGase F and Endo Hf. Both PNGase F and Endo Hf generated DR4-S protein of the same

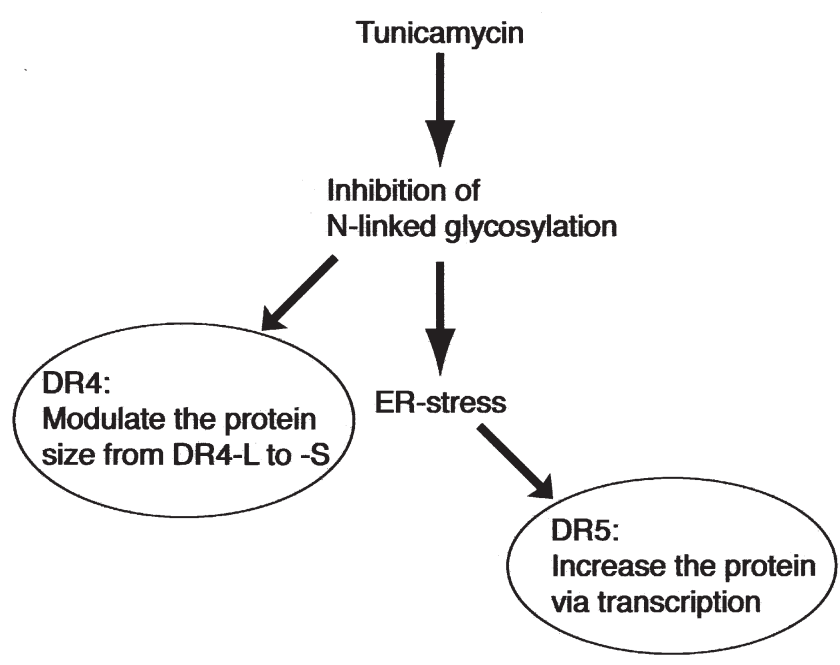

Figure 4. Tunicamycin differently regulates two pro-apoptotic receptors for TRAIL. Tunicamycin induces ER-stress following the inhibition of N-linked glycosylation. Tunicamycin increases DR5 protein via the regulation of transcription due to ER-stress. Furthermore, tunicamycin modulates the DR4 protein size from DR4-L to DR4-S due to inhibition of N-linked glycosylation.

size as that treated with tunicamycin (Fig. 3), whereas neither glycosydases changed DR5 protein. These results indicate that the modulation of DR4 protein by tunicamycin is due to the function of glycosylation inhibition.

\section{Discussion}

We show a novel regulation of DR4 protein in this study. Furthermore, we provide different regulations by one agent against two pro-apoptotic receptors for TRAIL, DR4 and 5 (Fig. 4). As for DR5, tunicamycin increased protein via transcription as we described previously (19). It is considered that this effect of tunicamycin is due to the induction of ER-stress, because other ER-stress inducers, thapsigargin and brefeldin A, also increased DR5 protein. On the other hand, for DR4, tunicamycin modulated the protein size without marked increase of DR4 protein. Our observations indicate that this effect depends on the inhibition of glycosylation but not on ER-stress, because the cleavage of glycoprotein by glycosydases caused the same change of DR4 protein and other ER-stress inducers did not change the size of DR4 protein. We also detected DR4-S protein in colon cancer cells treated with tunicamycin (data not shown), suggesting that this phenomenon is not specific to prostate cancer cells. Since a putative glycosylation site in amino acid sequences of DR4 protein has been reported (6), DR4 protein is probably directly glycosylated rather than processed by other glycoproteins.

Previously, we cloned the upstream promoter region of the DR5 gene (25) and demonstrated low similarity between DR4 and 5 promoter sequences (26), suggesting that the two gene expressions are regulated differently at the transcriptional level. Indeed, our and other groups reported agents which upregulated DR5 expression but did not change DR4 expression $(20,27)$. In the present study, we indicated that the regulation of DR4 and 5 is different at the protein level as well as the transcriptional level. 
$\mathrm{N}$-glycosylation regulates the functions of proteins, which are, for example, ligand binding against its receptor and enzyme activity $(15,16,28)$. We previously showed that DR5 up-regulation by tunicamycin sensitizes prostate cancer cells to TRAIL-induced apoptosis (19). Moreover, DR5 siRNA blocked this sensitization; however, DR4 siRNA did not block the sensitizing effect of tunicamycin on TRAIL-induced apoptosis (data not shown). In other cell lines, the modulation of DR4 protein by tunicamycin may affect the sensitivity against TRAIL. Recently, agonistic antibodies for DR4 and DR5 were developed to treat cancer patients $(11,12)$. DR4 antibody is involved in an on-going phase II trial (13). It could be of interest to examine whether DR4-L or $-\mathrm{S}$ protein influences the effect of the agonistic antibody.

It remains unclear how two different patterns of DR4 proteins affect the function of DR4. Further studies are needed to clarify this issue; however, we show here a novel mechanism regulating DR4 protein, and our observations in the present study may provide useful information for the elucidation of the full function of DR4 and for the clinical use of DR4 as a molecular target in cancer therapy.

\section{Acknowledgments}

This work was supported by the Japanese Ministry of Education, Culture, Sports, Science and Technology.

\section{References}

1. Wiley SR, Schooley K, Smolak PJ, et al: Identification and characterization of a new member of the TNF family that induces apoptosis. Immunity 3: 673-682, 1995

2. Pitti RM, Marsters SA, Ruppert S, Donahue CJ, Moore A and Ashkenazi A: Induction of apoptosis by Apo-2 ligand, a new member of the tumor necrosis factor cytokine family. J Biol Chem 271: 12687-12690, 1996.

3. Walczak H, Miller RE, Ariail K, et al: Tumoricidal activity of tumor necrosis factor-related apoptosis-inducing ligand in vivo. Nat Med 5: 157-163, 1999.

4. Ashkenazi A, Pai RC, Fong S, et al: Safety and antitumor activity of recombinant soluble Apo2 ligand. J Clin Invest 104: 155-162, 1999.

5. Lawrence D, Shahrokh Z, Marsters S, et al: Differential hepatocyte toxicity of recombinant Apo2L/TRAIL versions. Nat Med 7: 383-385, 2001

6. Pan G, O'Rourke K, Chinnaiyan AM, Gentz R, Ebner R, Ni J and Dixit VM: The receptor for the cytotoxic ligand TRAIL. Science 276: 111-113, 1997.

7. Pan G, Ni J, Wei Y-F, Yu G-L, Gentz R and Dixit VM: An antagonist decoy receptor and a death domain-containing receptor for TRAIL. Science 277: 815-818, 1997.

8. Sheridan JP, Marsters SA, Pitti RM, et al: Control of TRAILinduced apoptosis by a family of signaling and decoy receptors. Science 277: 818-821, 1997

9. Le Blanc HN and Ashkenazi A: Apo2L/TRAIL and its death and decoy receptors. Cell Death Differ 10: 66-75, 2003.
10. Sprick MR, Weigand MA, Rieser E, et al: FADD/MORT1 and caspase- 8 are recruited to TRAIL receptors 1 and 2 and are essential for apoptosis mediated by TRAIL receptor 2 . Immunity 12: 599-609, 2000.

11. Ichikawa K, Liu W, Zhao L, et al: Tumoricidal activity of a novel anti-human DR5 monoclonal antibody without hepatocyte cytotoxicity. Nat Med 7: 954-960, 2001.

12. Pukac L, Kanakaraj P, Humphreys R, et al: HGS-ETR1, a fully human TRAIL-receptor 1 monoclonal antibody, induces cell death in multiple tumour types in vitro and in vivo. Br J Cancer 92: 1430-1441, 2005.

13. Fesik SW: Promoting apoptosis as a strategy for cancer drug discovery. Nat Rev Cancer 5: 876-885, 2005.

14. Helenius A and Aebi M: Intracellular functions of N-linked glycans. Science 291: 2364-2369, 2001.

15. Wu JJ, Choi LE and Guidotti G: N-linked oligosaccharides affect the enzymatic activity of CD39: diverse interactions between seven N-linked glycosylation sites. Mol Biol Cell 16: 1661-1672, 2005.

16. Ding DX, Vera JC, Heaney ML and Golde DW: N-glycosylation of the human granulocyte-macrophage colony-stimulating factor receptor alpha subunit is essential for ligand binding and signal transduction. J Biol Chem 270: 24580-24584, 1995.

17. Shields RL, Lai J, Keck R, et al: Lack of fucose on human IgG1 $\mathrm{N}$-linked oligosaccharide improves binding to human $\mathrm{Fc} \gamma$ RIII and antibody-dependent cellular toxicity. J Biol Chem 277: 26733-26740, 2002

18. Elbein AD: Inhibitors of the biosynthesis and processing of N-linked oligosaccharide chains. Annu Rev Biochem 56: 497-534, 1987.

19. Shiraishi T, Yoshida T, Nakata S, et al: Tunicamycin enhances tumor necrosis factor-related apoptosis-inducing ligand-induced apoptosis in human prostate cancer cells. Cancer Res 65: 6364-6370, 2005

20. Nakata S, Yoshida T, Horinaka M, Shiraishi T, Wakada M and Sakai T: Histone deacetylase inhibitors up-regulate death receptor 5/TRAIL-R2, and sensitize apoptosis induced by TRAIL/APO2-L in human malignant tumor cells. Oncogene 23: 6261-6271, 2004

21. Wang S and El-Deiry WS: Inducible silencing of KILLER/DR5 in vivo promotes bioluminescent colon tumor xenograft growth and confers resistance to chemotherapeutic agent 5-fluorouracil. Cancer Res 64: 6666-6672, 2004.

22. Ren YG, Wagner KW, Knee DA, Aza-Blanc P, Nasoff M and Deveraux QL: Differential regulation of the TRAIL death receptors DR 4 and DR5 by the signal recognition particle. Mol Biol Cell 15: 5064-5074, 2004.

23. Sagara Y and Inesi G: Inhibition of the sarcoplasmic reticulum $\mathrm{Ca}^{2+}$ transport ATPase by thapsigargin at subnanomolar concentrations. J Biol Chem 266: 13503-13506, 1991.

24. Kain SR, Platt EJ, Brown KS, Black N and Firestone GL: Disruptions in intracellular membrane trafficking and structure preclude the glucocorticoid-dependent maturation of mouse mammary tumor virus proteins in rat hepatoma cells. J Biol Chem 267: 8128-8135, 1992.

25. Yoshida T, Maeda A, Tani N and Sakai T: Promoter structure and transcription initiation sites of the human death receptor 5/TRAIL-R2 gene. FEBS Lett 507: 381-385, 2001.

26. Yoshida $T$ and Sakai T: Promoter of TRAIL-R2 gene. Vitam Horm 67: 35-49, 2004.

27. Higuchi H, Bronk SF, Takikawa Y, Werneburg N, Takimoto R, El-Deiry W and Gores GJ: The bile acid glycochenodeoxycholate induces TRAIL-receptor 2/DR5 expression and apoptosis. J Biol Chem 276: 38610-38618, 2001.

28. Branza-Nichita N, Negroiu G, Petrescu AJ, et al: Mutations at critical $\mathrm{N}$-glycosylation sites reduce tyrosinase activity by altering folding and quality control. J. Biol Chem 275: 8169-8175, 2000. 